\title{
E-MQS - A new Downlink scheduler for Real-time flows in LTE network
}

\author{
Duy-Huy Nguyen, Hang Nguyen and Éric Renault \\ SAMOVAR, Télécom SudParis, CNRS, Université Paris-Saclay, 9 rue Charles Fourier - 91011 Evry Cedex \\ \{duy_huy.nguyen, hang.nguyen, eric.renault\}@ telecom-sudparis.eu
}

\begin{abstract}
This paper proposes a new scheduling scheme which based on the extended E-model, Channel- and QoS-Aware (known as E-MQS scheduler) for real-time traffics in LTE downlink direction. The real-time services (VoIP, Video, etc.) are very sensitive to network impairments such as delay, packet loss, jitter, etc. The proposed scheduling scheme is based on the extension of the E-model and the consideration of Maximum Queue Size (MQS) as a factor for the metric. Since this scheduling scheme considers Mean Opinion Score (MOS) values, thus, it gets higher user perception. The simulation results show that the proposed scheme has the performance which not only satisfies QoS requirements of real-time services but also outperforms the Frame Level Scheduler (FLS), Modified Largest Weighted Delay First (M-LWDF) and Exponential/Proportional Fair (EXP/PF) schedulers in terms of delay, cell throughput, Fairness Index (FI) and Spectral Efficiency (SE), especially for Video flow. Our proposed scheduler also significantly improves the Packet Loss Rate (PLR) in comparison with the M-LWDF and EXP/PF schedulers for both VoIP and Video flows. The performance evaluation is compared in terms of Delay, PLR, Throughput, FI and SE for FLS, M-LWDF, EXP/PF schedulers and our proposed one.
\end{abstract}

Index Terms-Scheduling algorithm, Resource allocation, Realtime services, LTE, QoS, E-model, QoE, MQS.

\section{INTRODUCTION}

The Long Term Evolution (LTE) is developed by the Third Generation Partnership Project (3GPP) [1]. It is a mobile network which has high data rate, low delay and fully packetbased. It means to improve the capability of legacy system by increasing data rates and extending superior Quality of Service (QoS) for various multimedia applications. Basic components of LTE network include a powerful eNodeB (eNB) station and several User equipments (UEs) in addition to a gateway [2]. The eNB station combines with core network through several standard complicated protocols. Basic packet scheduling is carried out by the network operator in both UE and eNB station for both uplink as well as downlink. However, according to the 3GPP, there are no firm specifications for scheduling technique in LTE network. One of the most important modules of packet scheduling is Radio Resource Management (RRM) which decides users that would transmit their data on the air interface. The packet scheduling should integrate fairness in terms of throughput as well as the service policies to which users subscribe [3].

In this paper, we propose a new downlink scheduling scheme for real-time communications in LTE network with the consideration of user perception. There are several papers which mentioned it. Specifically, authors in [4], [5] proposed a new Quality of Experience (QoE)-driven LTE Downlink Scheduling for VoIP Application that is based on QoE min (i.e. MOS score at least equals 3.5 for VoIP Application) and they optimize number of users to access a cell. In [6], the authors a cross-layer design scheme that jointly optimises three different layers of wireless protocol stack, namely Application, Medium Access Control (MAC) and Physical layers. The purpose of this paper is to maximize network resource utilization and user-perceived quality of service (also called Quality of Experience - QoE). These papers proposed a new LTE Downlink Scheduler but not based on the E-model. The paper which is closest to our paper described in [7]. In this paper, the authors proposed a new scheduling scheme for VoIP service in LTE networks by using the user satisfaction as a metric for their scheduler. The authors used the E-model to predict user perception via MOS score, and then this factor used in the metric for scheduling decision. However, in this paper, authors did not consider the impact of network jitter for E-model. In addition, in the metric of the scheduler, there is no the presence of the MQS, and the authors evaluated only for VoIP traffic. In our paper, we extend the idea in [7] by extending the E-model and propose to consider the MQS as an essential and effective factor for the metric. We used the extended E-model to predict the MOS score and use this score as a main factor in the metric. Besides, we see that, the MQS factor has significant effects on the system performance. In the LTE-Sim [8], this factor is fixed equal to 0. This means the MQS is infinite. So that, in the scheduling process, the MQS is not considered. However, in fact, the MQS should be a finite value because if the MQS value is infinite then the delay will increase and the congestion could be increased. Therefore, the MQS needs to be considered as a essential factor in the metric of the scheduling algorithms.

The rest of this paper is organized as follows: Overview of the system model is described in section II. In section III, we present the proposed scheduling scheme. The simulation results and performance evaluation of the proposed scheduler are analysed in section IV. The conclusion and future work is represented in section $\mathrm{V}$.

\section{THE SYSTEM MODEL}

\section{A. The E-model}

E-model is a computational model developed and standardized by ITU-T [9]. It is used to estimate the MOS for narrow 
band audio quality. The output of the model is R-factor. The values of this $\mathrm{R}$-factor in range of $0-100$ where 100 is the best and 0 is the worst quality. And then, it is mapped to the corresponding MOS value. The standard R-factor in the E-model is defined as follows:

$$
R=R_{0}-I_{s}-I_{d}-I_{e f}+A
$$

In which: $R_{0}$ : The basic signal-to-noise ratio which consists of noise sources such as circuit and room noise. In this model, its value is set to 94.2. $I_{s}$ : The simultaneous impairment factor, it is the sum of all impairments which may occur more or less simultaneously with the voice transmission. In this model, the default value is set to $0 . I_{d}$ : The delay impairment factor, representing all impairments due to delay of voice signals. $I_{e f}$ : The equipment impairment factor, capturing the effect of signal distortion due to low bit rates of the codec and packet losses of random distribution. $A$ : The advantage factor, capturing the fact that some users can accept a reduction of quality due to the mobility of cellular networks. In this model, this factor is set to 0 .

In above factors, $I_{d}$ and $I_{e f}$ are affected by end-to-end delay and packet loss, respectively, while $R_{0}$ and $I_{s}$ do not depend on network performance. The R-factor is then translated into the MOS as follows [9]:

$$
M O S=\left\{\begin{array}{l}
1, \text { if } R<0 \\
1+0.035 \times R+7 \times 10^{-6} \times R \times(R-60) \times \\
(100-R), \text { if } 0 \leq R \leq 100 \\
4.5, \text { otherwise }
\end{array}\right.
$$

After setting the default values for the E-model, Equation (1) can be rewritten as follows:

$$
R=94.2-I_{d}-I_{e f}
$$

We see that, when voice packet transmitted over an IP network, is is affected by many network impairments such as PLR, delay, jitter, etc. In the E-model, there is no presence of network jitter. In order to improve use satisfaction, we propose to add the $I_{j}$ factor to the E-model. Hence, the E-model can be described as the following formula:

$$
R=94.2-I_{d}-I_{e f}-I_{j}
$$

Equation (4) shows that the R-factor depends on end-to-end delay $\left(I_{d}\right)$, total loss probability $\left(I_{e f}\right)$, and network jitter $\left(I_{j}\right)$. Hence, in order to compute the R-factor, we must to count these factors. The $I_{d}$ is a factor which is affected by end-toend delay and is calculated as follows [10]:

$$
I_{d}=0.024 \times d+0.11 \times(d-177.3) \times H(d-177.3)
$$

In which: $H(x)$ is the Heavyside function:

$$
H(x)=\left\{\begin{array}{l}
0, \text { if } x<0 \\
1, \text { otherwise }
\end{array}\right.
$$

In equation (5), $d$ represents the total end-to-end delay (or mouth-to-ear delay) of speech packet. It can be calculated via some functions in LTE-Sim tool. The $I_{e f}$ is determined according to packet loss. In order to compute this factor, we use the equation in [7] as follows:

$$
I_{e f}=\lambda_{1}+\lambda_{2} \times \ln \left(1+\lambda_{3} \times e_{l}\right)
$$

Where: The $\lambda_{1}$ represents the voice quality impairment factor caused by the encoder, $\lambda_{2}$ and $\lambda_{3}$ represent the effect of loss on voice quality for a given codec. Such that, these factors depend on the voice codec used. In this study, we use LTE-Sim [8] to simulate. This simulation tool supports only G.729 codec, thus, for this codec, the factors above has values as follows: $\lambda_{1}=11, \lambda_{1}=40, \lambda_{3}=10$. While $e_{l}$ is the total loss probability (consisting of network and buffer layout) which has the value in range of $0 . .1$. This factor is computed directly via some functions in the LTE-Sim tool.

The $I_{j}$ represents the impacts of network jitter to voice quality. It also depends on the voice codec. In this paper, we use the method proposed in [11] as follows:

$$
I_{j}=C_{1} \times H^{2}+C_{2} \times H+C_{3}+C 4 \times e^{-T / K}
$$

In which: $C_{1}, C_{2}, C_{3}, C_{4}$ are coefficients, $\mathrm{K}$ is time instant. These factors depend on the voice codec, for the G.729 codec, these factors have the values as follows: $C_{1}=-15.5, C_{2}=$ $33.5, C_{3}=4.4, C_{4}=13.6, K=30$. The factor of $\mathrm{T}$ is the fixed buffer size of the voice codec. For the G.729 codec, the packet size is $20 \mathrm{~ms}$, thus, we select $\mathrm{T}=40 \mathrm{~ms}$. The $H$ is a factor of Pareto distribution and in range of 0.55 to 0.9 . According to [11], the MOS drops when $H$ increases, thus, in this study, we select $\mathrm{H}=0.6$ for the simulation.

The final expression of the R-factor when utilizing the G.729 codec is described in Equation (9).

$$
\begin{aligned}
& R=64.28-[0.024 \times d+0.11 \times(d-177.3) \times \\
& H(d-177.3)]-40 \times \ln \left(1+10 \times e_{l}\right)-13.6 \times e^{-4 / 3}
\end{aligned}
$$

The R-factor is then mapped to the MOS via equations (2) MOS is one of the important factors for the metric in our scheduler.

\section{B. The correlated Scheduling algorithms}

We assume that the metric assigned to the stream $i$ on $j$-th sub-channel is noted by $w_{i, j}$. In order obtain the metric, the scheduler usually need to know the average transmission rate $\left(\bar{R}_{i}\right)$ of flow $i$, and the flow rate available to the UE on the $j$-th sub-channel. In particular, at each TTI, the estimate $\bar{R}_{i}$ is given by [8]:

$$
\bar{R}_{i}(k)=0.8 \times \bar{R}_{i}(k-1)+0.2 \times \bar{r}_{i}(k)
$$

Where: $\bar{R}_{i}(k-1)$ : The average transmission data rate estimating at the $(k-1)$-th TTI. $\bar{r}_{i}(k)$ : The rate allocated to $i$-th flow during the $k$-th TTI.

In the following subsections, we will describe the metric of several well-known scheduling algorithms which are related to our proposed scheduling scheme including: FLS, M-LWDF, and EXP/PF schedulers. We select these scheduling because they perform well and are suitable for real-time services. 
1) The FLS scheduler: FLS is a two-level scheduling algorithm which are called upper level and lower level. These levels are distinct and communicate with each other to dynamically allocate the RBs to the users. At the upper level, a resource allocation method (called FLS) which uses a Discrete-Time (D-T) linear control theory is performed. FLS defines the amount of data that each real-time source should transmit within a single frame to meet its delay constraint. At the lower level, the algorithm uses Proportional Fair (PF) method to allocate RBs to the users at each TTI with considering the bandwidth requirements of FLS to ensure a good level of fairness among multimedia flows. Also at this layer, the scheduler determines number of TTIs/RBs via that each Real-time source will send its packets. In order to calculate the amount of data transmitted, the FLS scheduler uses the following formula:

$$
V_{i}(k)=h_{i}(k) * q_{i}(k)
$$

In which: $V_{i}(k)$ is the amount of the data transmitted by the flow $i$ in LTE frame $k$, '*' operator is the discrete time convolution, $q_{i}(k)$ is the queue level. It can be said that, $V_{i}(k)$ is computed by filtering the signal $q_{i}(k)$ via a time-invariant linear filter with pulse response $h_{i}(k)$.

2) The M-LWDF scheduler: M-LWDF scheduling algorithm is used to support multiple real-time services in CDMAHDR systems [12]. For each real-time flow, by considering the maximum time $\tau_{i}$, the probability is defined as the maximum probability $\delta_{i}$ which is the time of the first packet of the queue exceeds the fixed maximum time $D_{H O L, i}$. In this algorithm, the metrics for real-time and non real-time services are different. In order to offer priority to real-time flows, the metric was given as follows:

$$
w_{i, j}=\alpha_{i} \times D_{H O L, i} \times \frac{r_{i, j}}{R_{i}}
$$

Where:

- $r_{i, j}$ : The rate assigned to $i$-th flow during the $k$-th TTI

- $\bar{R}_{i}$ : The average transmission data rate estimating

- $\alpha_{i}$ : A factor and is given by: $\alpha_{i}=-\frac{\log \left(\delta_{i}\right)}{\tau_{i}}$

3) The EXP/PF scheduler: EXP/PF is a scheduling algorithm which supports multimedia applications in an adaptive modulation and coding and time division multiplexing (AMC/TDM) system [13]. The main purpose of this scheduling algorithm is to enhance the priority for the real-time flows by adding the average fixed maximum time of all active real-time flows. For the real-time services, they receive the increased priorities when their HOL packet delays are approaching the delay deadline. The metric of EXP/PF is calculated as follows:

$$
w_{i, j}=\exp \left(\frac{\alpha_{i} \times D_{H O L, i}-X}{a+\sqrt{X}}\right) \times \frac{r_{i, j}}{R_{i}}
$$

Where $\mathrm{X}$ is given by: $X=\frac{1}{N_{r t}} \sum \times \alpha_{i} \times D_{H O L, i}$, with $N_{r t}$ is the number of active real-time flows in the downlink direction.

The remaining parameters are similar to the descriptions above.

\section{THE PROPOSED SCHEDULING SCHEME}

In our proposed scheduling scheme, we consider the characteristics of the real-time services such as VoIP, Video, etc. These services are sensitive to packet loss and delay, thus, scheduling process should consider various factors. In the related scheduling algorithms above, the authors almost focused on Head of Line packet delay, virtual token length besides other factors such as $\alpha_{i}, r_{i, j}$ and $\bar{R}_{i}$. MOS is a parameter which represents user perception, thus, it should appear in the metric of scheduling algorithms. The higher MOS, the higher user satisfaction. MOS needs be automatically calculated at the receiver and is sent to the eNodeB via feedback technique. For the MQS, according to our knowledge, there are no articles which mention about it. We think that, this factor has strong effects on the system performance. In the LTE-Sim [8], this factor is fixed equal to 0 . This means the MQS is infinite. Hence the MQS is not considered in the scheduling process. However, in fact, the MQS should be finite. If the MQS value is infinite then the delay will increase and the congestion could be thus increased. Therefore, the MQS should be considered as a necessary factor in the metric of the scheduling algorithms.

The main idea of our scheduling algorithm is the consideration of user satisfaction (MOS) and the MQS factor (called also $Q_{i, \max }$ ) included into the metric of the scheduling algorithm. This means the higher MOS and the lower $\left(Q_{i, \max }-Q_{i}\right)$ values, the higher priority for the UE. The fixed maximum time $D_{H O L, i}$ and the maximum probability $\delta_{i}$ are included in the Equation (9) to calculate the factors of $I_{d}, I_{e f}$, respectively. The metric in our scheduling scheme for the real-time services is defined as follows:

$$
w_{i, j}=\frac{\operatorname{MOS}_{i} \times\left(Q_{i, \max }-Q_{i}\right)}{\tau_{i}} \times \frac{r_{i, j}}{\bar{R}_{i}}
$$

Where:

- $Q_{i}, \tau_{i}, r_{i, j}$ and $\bar{R}_{i}$ have the same significances in the previous formulas.

- $Q_{i, \max }$ : The MQS of the user $i$. This value can be obtained in bytes via some functions in LTE-Sim [8].

For the non real-time services, we propose to use the method in the PF scheduler [14]. The $w_{i, j}$ is a priority matrix for each $R B_{j}$ is assigned to $U E_{i}$. It is calculated based on the MOS, the remaining queue size $\left(Q_{i, \max }-Q_{i}\right)$, the maximum time $\tau_{i}$ and the channel condition. MOS is computed at the receiver and is feedbacked to the eNodeB in order to make the scheduling decision of UE. MOS included in the metric will fully exploit the user perception.

\section{Simulation environment and Performance EVALUATION}

\section{A. Simulation parameters}

The basic parameters used in the simulation are represented in the Table I.

\section{B. Performance evaluation}

In order to evaluate the performance of our scheduling scheme, we use the LTE-Sim [8] to simulate the proposed 
TABLE I

SIMULATION PARAMETERS

\begin{tabular}{|l|l|}
\hline Simulation Parameters & \multicolumn{1}{|c|}{ Values } \\
\hline Simulation duration & $100 \mathrm{~s}$ \\
\hline Frame structure & FDD \\
\hline Cell radius & $1 \mathrm{~km}$ \\
\hline Bandwidth & $10 \mathrm{MHz}$ \\
\hline Video bit-rate & $242 \mathrm{kbps}$ \\
\hline VoIP bit-rate & $8.4 \mathrm{kbps}$ \\
\hline User speed & $3 \mathrm{~km} / \mathrm{h}$ \\
\hline Number of users & $5,10,15,20,25 \mathrm{UEs}$ \\
\hline Maximum delay & $0.1 \mathrm{~s}$ \\
\hline MQS & $10^{5}$ bytes \\
\hline Traffic model & VoIP and Video \\
\hline
\end{tabular}

scheduler with the other schedulers including the FLS, MLWDF and EXP/PF. The performance evaluation is compared in terms of delay, PLR, cell throughput, FI and SE. The analysis of the simulation results are represented in the following subsection.

1) Delay: End-to-end delay (called one-way delay) is the time required for a packet to be transmitted from source to destination in the network. Figure 1(a) illustrates the delay of VoIP flow. It is clear that, the M-LWDF and EXP/PF scheduler has the same lowest delay. Our proposed scheduler (E-MQS) has the delay which is not significantly higher than the MLWDF and EXP/PF schedulers and it outperforms the FLS scheduler.

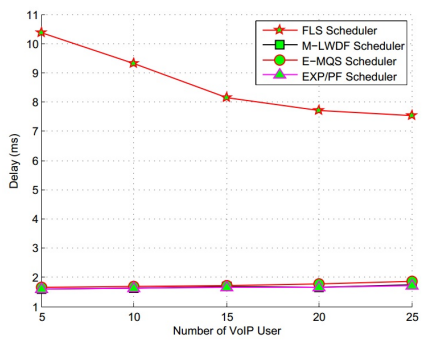

(a) Delay vs number of VoIP user

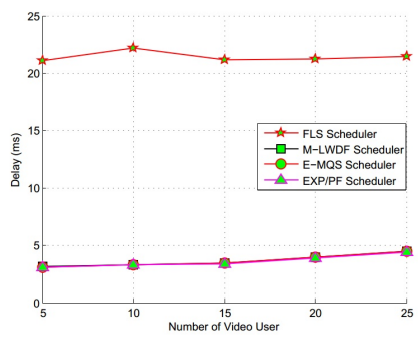

(b) Delay vs number of Video user
Fig. 1. Effects of Delay vs VoIP and Video users

For the video flow, all schedulers have the delay which slightly increase when the UEs increase as shown in Figure 1(b). Our proposed scheduler, the M-LWDF and EXP/PF schedulers have nearly the same delay. The FLS has the delay which is higher than the other schedulers. However, all the schedulers have the good end-to-end delay when the number of UE increases equal to 25 . It can be included that all the schedulers are also very suitable for video flow.

2) Packet Loss Rate: PLR shows the failure of one or more transmitted packets to reach their destination across a network. Figure 2(a) represents the PLR of VoIP flow. When we set the MQS equal to $10^{5}$ bytes (this factor is set equal to 0 as default in the LTE-Sim), for the VoIP flow, all schedulers have the decreased PLR when the number of UE increases. Normally, the PLR increases when the number of UE increases, thus, this case is quite special and it represents the unstableness of a real system. As shown in the Figure 2(a), all the schedulers have the PLR which are under $1 \%$ while the FLS has the lowest
PLR. The E-MQS scheduler has the second position while the M-LWDF has the highest PLR.

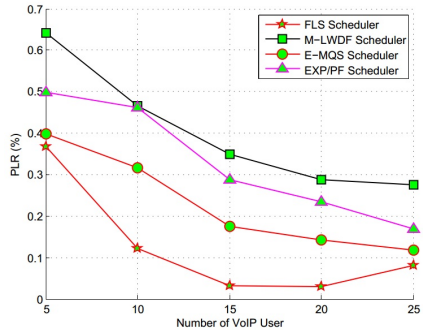

(a) PLR vs number of VoIP user

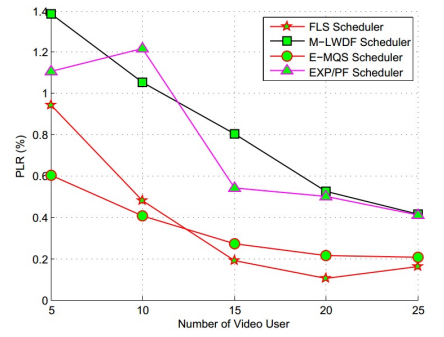

(b) PLR vs number of Video user
Fig. 2. Effects of PLR vs VoIP and Video users

For the Video flow, as shown in the Figure 2(b), also as for VoIP flow, the PLR decreases when the number of UE increases. However, for all the schedulers, the PLR under $1 \%$ when the number of UE more than 12. For the FLS and EMQS schedulers, the PLR less than $1 \%$ for all number of UE. For the number of UE from 5 to 12 , the proposed scheduler has the lowest PLR, when the number of UE greater than 12, the FLS scheduler has the lowest PLR and the E-MQS keeps the second position. In general, the FLS scheduler has the lowest PLR and the M-LWDF has the highest PLR.

3) Cell throughput: As shown in Figure 3(a), for the VoIP flow, the cell throughput of all the schedulers increases when the number of UE increases. The proposed scheduler always has the cell throughput in the top of 2 highest schedulers for all cases of the number of the UE. This means the proposed scheduler is very suitable for VoIP flow.

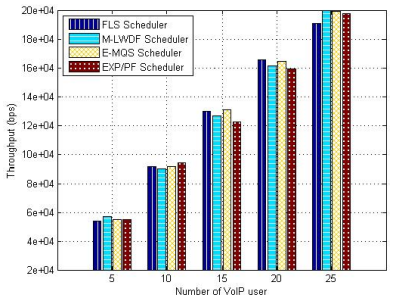

(a) Throughput vs number of VoIP user

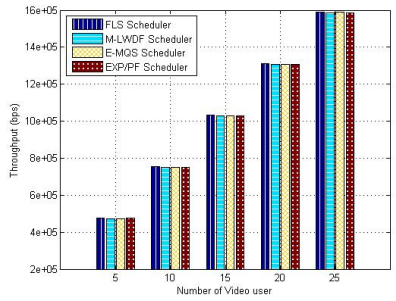

(b) Throughput vs number of Video user
Fig. 3. Effects of Throughput vs VoIP and Video users

For the Video flow, in the proposed scenario, all schedulers have the near values of cell throughput for all cases of the number of UE as shown on Figure 3(b). This is very interesting case. This concludes that all the schedulers are very conformable to Video flow. Also as VoIP flow, the cell throughput increases when the number of UE increases. This is very good for real-time flows such as VoIP, Video, etc.

4) Fairness index: For the VoIP flow as shown on Figure 4(a), the FIs of all schedulers are not stable when the number of UE increases. However, all these schedulers have the high FIs. The proposed scheduler has the highest FI when the number of UE equals 5, 15, and 20 and obtains the lowest FIs in the case of the number of UE equals 10, and 20. In general, the FLS has the best FI for VoIP flow. 


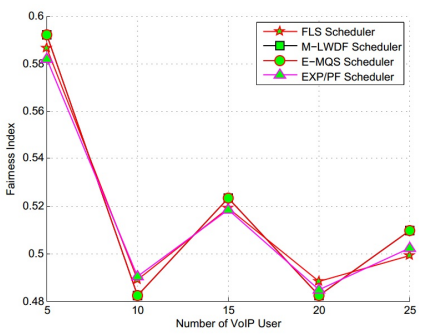

(a) FI vs number of VoIP user

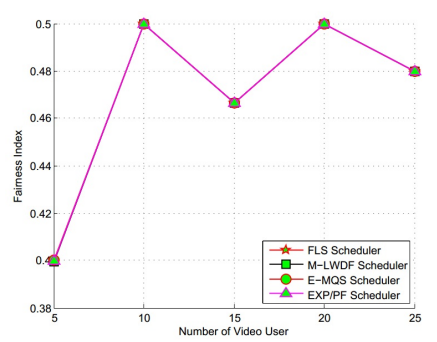

(b) FI vs number of Video user
Fig. 4. Effects of Fairness Index vs VoIP and Video users

Figure 4(b) shows the FI of the schedulers. The FI changes when the number of UE increases. It is clear that, the FI of all the schedulers is nearly the same. This is also a very interesting case in the proposed scenario. This not usually happens. This demonstrates that all the schedulers consider the FI is very important in the metric of them.

For the FI, it can be conclusive that the FLS scheduler has the best performance. This is due to at the lower layer of this scheduler uses PF algorithm, thus, it ensures the good grade of fairness among multimedia flows. The FI is high which demonstrates that the cell-edge users have been guaranteed the minimum performance. Hence these users can be served when they move to the edge of the cell.

5) Spectral efficiency: The successful usage of radio resources is a basic purpose of scheduling algorithms. The SE is seen as the performance measurements for the entire cell. As shown on Figure 5, the SE increases when the number of UE increases. In almost case of the number of UE, the proposed and the FLS schedulers have the same highest SE. However, there is no the difference among schedulers.

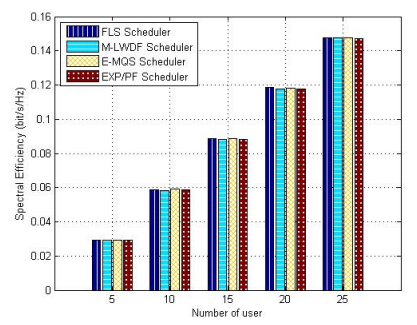

Fig. 5. Spectral efficiency vs number of user

\section{CONCLUSION}

In this paper, we propose a new Channel-, QoS- and QoEAware scheduling scheme for the downlink direction in LTE network. The main idea in the proposed scheduler is the consideration of the MOS and the MQS factors into the metric in the proposed scheduler. The metric is based on the user perception (MOS), the remaining queue size, the fixed maximum time, and the channel condition. The simulation results show that the proposed scheduler not only meets QoS requirements for real-time services but also outperforms the FLS, M-LWDF, EXP/PF schedulers in terms of delay, cell throughput, FI and SE, especially for Video flow. For the PLR in the case of VoIP flow, the proposed scheduler has the performance not as well as the FLS scheduler. For the FI in the case of VoIP flow, the proposed scheduler does not have the stable performance it obtains or the highest either the lowest FI values. The proposed scheduler also significantly improves the delay in comparison with the M-LWDF and EXP/PF when the number of UE more than than 12 for Video flow. It can be said that when considering the MOS and the MQS as factors for the metric in the proposed scheduler, the system performance has been improved significantly, specially for Video flow. Through all simulation results, it can be said that, the FLS has the best performance, and the proposed scheduler keeps the second position in scheduling for both VoIP and Video flows. So that, the proposed scheduler is very suitable for real-time services such as VoIP, Video, etc. for the downlink direction in LTE system.

In this study, we have not yet considered the presence of best-effort flow. They might bring new directions to evaluate the proposed scheduler in heterogeneous traffic in LTE network.

\section{REFERENCES}

[1] 3GPP, http://www.3gpp.org

[2] S. Ali and M. Zeeshan, "A utility based resource allocation scheme with delay scheduler for lte service-class support," in Wireless Communications and Networking Conference (WCNC), 2012 IEEE. IEEE, 2012, pp. $1450-1455$.

[3] M. Alasti, B. Neekzad, J. Hui, and R. Vannithamby, "Quality of service in wimax and le networks [topics in wireless communications]," Communications Magazine, IEEE, vol. 48, no. 5, pp. 104-111, 2010.

[4] A. Alfayly, I. Mkwawa, L. Sun, and E. Ifeachor, "Qoe-based performance evaluation of scheduling algorithms over lte," in Globecom Workshops (GC Wkshps), 2012 IEEE. IEEE, 2012, pp. 1362-1366.

[5] A. Alfayly, I.-H. Mkwawa, L. Sun, and E. Ifeachor, "Qoe-driven lte downlink scheduling for voip application," in Consumer Communications and Networking Conference (CCNC), 2015 12th Annual IEEE. IEEE, 2015, pp. 603-604.

[6] M. Nasimi, M. Kousha, and F. Hashim, "Qoe-oriented cross-layer downlink scheduling for heterogeneous traffics in lte networks," in Communications (MICC), 2013 IEEE Malaysia International Conference on. IEEE, 2013, pp. 292-297.

[7] M. S. Mushtaq, B. Augustin, and A. Mellouk, "Qoe-based lte downlink scheduler for voip," in Wireless Communications and Networking Conference (WCNC), 2014 IEEE. IEEE, 2014, pp. 2190-2195.

[8] G. Piro, L. A. Grieco, G. Boggia, F. Capozzi, and P. Camarda, "Simulating lte cellular systems: an open-source framework," Vehicular Technology, IEEE Transactions on, vol. 60, no. 2, pp. 498-513, 2011.

[9] ITU-T, "Itu-t recommendation g.107: The e-model, a computational model for use in transmission planning," International Telecommunication Union, Tech. Rep., Dec. 1998.

[10] C. Olariu, M. O. Foghlu, P. Perry, and L. Murphy, "Voip quality monitoring in lte femtocells," in Integrated Network Management (IM), 2011 IFIP/IEEE International Symposium on. IEEE, 2011, pp. 501508.

[11] L. Ding and R. A. Goubran, "Speech quality prediction in voip using the extended e-model," in Global Telecommunications Conference, 2003. GLOBECOM'03. IEEE, vol. 7. IEEE, 2003, pp. 3974-3978.

[12] P. Ameigeiras, J. Wigard, and P. Mogensen, "Performance of the mlwdf scheduling algorithm for streaming services in hsdpa," in Vehicular technology conference, 2004. VTC2004-Fall. 2004 IEEE 60th, vol. 2. IEEE, 2004, pp. 999-1003.

[13] J.-H. Rhee, J. M. Holtzman, and D.-K. Kim, "Scheduling of real/nonreal time services: adaptive exp/pf algorithm," in Vehicular Technology Conference, 2003. VTC 2003-Spring. The 57th IEEE Semiannual, vol. 1. IEEE, 2003, pp. 462-466.

[14] A. Jalali, R. Padovani, and R. Pankaj, "Data throughput of cdma-hdr a high efficiency-high data rate personal communication wireless system," in Vehicular technology conference proceedings, 2000. VTC 2000-Spring Tokyo. 2000 IEEE 51st, vol. 3. IEEE, 2000, pp. 1854-1858. 